\title{
A review of GOLPH2, an oncogenic protein and novel therapeutic options for GOLPH2 driven tumours
}

\author{
Yang Liu ${ }^{1}$, Heike Liewen ${ }^{2}$, Norbert Markuly ${ }^{2}$ and Frank Stenner ${ }^{1 *}$ \\ ${ }^{1}$ Medical Oncology, University Hospital Basel and Laboratory Cancer Immunology, Department Biomedicine, University Basel, Petersgraben 4, 4031 Basel, \\ Switzerland \\ ${ }^{2}$ Cureab GmbH, Benkenstrasse 254c, Technologiezentrum, 4108 Witterswil, Switzerland
}

\begin{abstract}
GOLPH2 (Golgi Phosphoprotein 2) is a protein with pro-oncogenic properties that has currently been identified as a prime target for tumour therapy. Its involvement in key oncogenic pathways like EGFR, mTOR/AKT, PI3K makes it a good candidate for therapeutic intervention. Several research groups have consistently reported that GOLPH2, when expressed at high levels leads to epithelial to mesenchymal transition (EMT), increased cell proliferation, migration and metastasis.

Not amazingly, it appears that many tumours exploit GOLPH2 for their advantage. Stopping GOLPH2's detrimental effects would mean impairing tumour progression on various levels of its development. Therefore, inhibition of GOLPH2, a protein contributing to important hallmarks of cancer deserves the attention of researchers and drug developing stakeholders. However, its mainly intracellular localisation and the lack of domains that could possibly be interfered with by small molecules, have led to the conclusion that GOLPH2 is an un-targetable molecule.

Here, we summarize the current knowledge of this multifunctional protein and describe possibilities to pharmacologically intervene to ameliorate its overshooting function in malignant diseases. Novel approaches like viral interventions or specific antibodies could soon result in a substantial therapeutic improvement for cancers with underlying GOLPH2 pathologies.
\end{abstract}

\section{Introduction}

The role of GOLPH2 as an oncogenic protein has been established by a large body of work showing that GOLPH2 over-expression increases cell proliferation, migration and metastasis. In addition, GOLPH2 is involved in epithelial to mesenchymal transition [1-3] a pre-requisite for the above properties. In this review we highlight important findings in this field and discuss possibilities to target GOLPH2 for cancer therapy.

Golgi Phosphoprotein 2 (GOLPH2, gp73, GOLM1) is a single pass type II transmembrane protein and it belongs to the GOLM1/CASC4 family (www.uniprot.org). Its exact function is unknown, but it has been described as a cellular response protein to viral infections and is associated with various cancers. GOLPH2's protein organization is shown in the following simplified scheme (Figure 1).

GOLPH2's short cytoplasmic tail (CT: amino acids 1-12) and its transmembrane domain (TM: aa 13-35) are important for the localization to the Golgi [4]. The intra-lumenal part, the major component of the protein with 366 amino acids contains a furin cleavage site near to the membrane (dashed line at aa 55). Proteolytic cleavage allows the release of intra-lumenal's main part called soluble GOLPH2

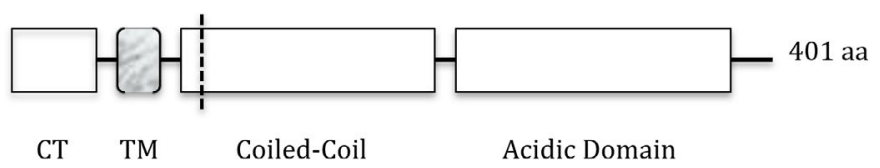

Figure 1. GOLPH2's protein organization is shown in a simplified scheme
(sGOLPH2). sGOLPH2 (aa 56-401) consists of two continuous alpha helices. The second helix near the C-terminus is enriched with acidic residues (acidic domain).

Soluble GOLPH2 is a very stable protein and can be measured in supernatants of cultured cells, serum, urine and other body fluids. The exact instances under which GOLPH2 is processed have not been clarified to date, but increased cell proliferation seems to promote GOLPH2 cleavage and results in higher extracellular concentrations of the soluble form [5].

GOLPH2 appears to have a variety of different functions what has precluded a concise determination of its physiologic role until today. GOLPH2's expression is tightly regulated, reports of several miRNAs involved in its post-transcriptional regulation have been published indicating the importance of homeostatic GOLPH2 levels.

But it has also been noted that GOLPH2's function in cell physiology appears to be non-vital for development and survival. The published knockout mouse is viable and displays only a modest phenotype, namely a higher susceptibility to LPS challenge compared to wildtype littermates [6].

${ }^{\star}$ Correspondence to: Frank Stenner, Medical Oncology, University Hospital Basel and Laboratory Cancer Immunology, Department Biomedicine, University Basel, Petersgraben 4, 4031 Basel, Switzerland, E-mail: frank.stenner@usb.ch

Key words: GOLPH2, Golm1, GP73, EGFR, PDGFR, AKT, mTOR, ERK, EMT

Received: October 19, 2019; Accepted: November 01, 2019; Published: November 06, 2019 
A truncated GOLPH2 however, seems to impair normal renal and hepatic development. Wright and colleagues have described a transgenic mouse that carries a c-terminally truncated GOLPH2 protein. This transgene lacking the acidic domain leads to a decreased life span and notable hepato-renal pathology [7].

Summarizing these findings, it can be stated that GOLPH2 appears to be important but during development and under normal conditions it is functionally replaceable in the organism.

GOLPH2 has been shown to modulate cellular responses to infections, for example in viral diseases like hepatitis [8]. While overexpression of GOLPH 2 has been observed in many cancers, highest concentrations of its soluble form have been measured in hepatocellular carcinoma (HCC) [9] and only to a much lesser extend in other cancers, e.g. lung adenocarcinoma [10], despite being overexpressed in both tumours. This difference could be attributed to the lumenal secretion of GOLPH2 by tumour cells that would foster GOLPH2 concentration in the lumenal space rather than in the blood circulation. The high serum content of GOLPH2 in HCC would then be the exception from this rule and might be explained by an intrahepatic cholestasis precluding its lumenal excretion.

\section{GOLPH2, a biomarker for malignant diseases}

Techniques of GOLPH2 quantification have evolved in recent years from time-consuming Western Blotting (WB) [11]. via Enzyme linked Immuno Sorband Assays (ELISA) [11-15] to particle based unpretentious bioassays. Quantification of GOLPH2 expression in tumour tissue by immunohistochemistry (IHC), a routine clinical technique, paved the way for exploration of GOLPH2 expression in solid tumours like HCC, prostate [16] and gastric cancer [17], lung adeno carcinoma [18,19], glioblastoma [20] and melanoma [21].

HCC remained the pivotal disease in which GOLPH2 was recognized as a potential biomarker. Several groups have shown that it was at least as good as AFP, the most often used tumour marker in this disease and that combination of GOLPH 2 and AFP could outperform each of the markers alone $[9,22,23]$. It has to be mentioned that there is criticism in terms of the value of GOLPH2 as a biomarker in HCC [24]. Here, the authors regard the selectivity for distinction of cirrhosis and carcinoma as insufficient. As of today, serum GOLPH2 has not become a routine tumour marker in the diagnostics of HCC. Besides HCC, GOLPH2 has drawn considerable interest in adenocarcinomas of the lung and prostate. Several groups have shown, that GOLPH2 is a valuable prostate cancer marker in tissue, blood and urine of patients with localized prostate cancer [16,25-27]. In a study that tested a multiplexed assay GOLPH2 compared favourably with PSA. There, GOLPH2 had a ROC with an AUC of 0.664 compared to an AUC of 0.508 for PSA [28]. Regarding tumours of the lung GOLPH2 has been implicated in adenocarcinomas. There the protein is overexpressed and found in moderately elevated serum levels. However, in squamous cell carcinomas no significant overexpression was found [10]. In another study [29] the authors used data from the Gene Expression Omnibus and The Cancer Genome Atlas (TCGA) and showed overexpression of GOLPH2 in lung adenocarcinomas as well as squamous cell carcinomas (SCC). However, while relapse free survival and OS was worse in patients with GOLPH2 positive adenocarcinomas, no difference was noted for patients with SCC. Other epithelial carcinomas show very similar pattern when GOLPH2 is overexpressed and disease related. These include oral SCC [30] and gastric carcinomas [17], where high GOLPH2 levels were associated with worse outcome. In summary, there is a broad basis for the use of GOLPH2 as biomarker in different solid tumours.

\section{Regulation of GOLPH2 (miRNA-143, miRNA-145, miRNA-200)}

miRN As have been involved in gene regulation and aberrancies in their levels can lead to pathognomonic circumstances. Three miRNAs were found to be involved in the transcriptional regulation of GOLPH2 and are thus of interest. miR-143 was shown to be downregulated in cervical cancer [31]. This downregulation corresponded to an increased GOLPH2 level promoting invasiveness and a pro-metastatic phenotype of the respective cancers. A transcriptional search revealed GOLPH2 to be a target sequence of miR-143 and experiments utilizing inhibitors and mimics of this miRNA gave proof that the negative correlation of this miRNA and GOLPH2 levels corresponded to the described phenotype.

Gai X. et al. showed that miR-145 is suppressed by the mammalian target of rapamycin (mTOR) and this leads to increased GOLPH2 expression [32]. The decrease of miR-145 promotes hepatocellular cancer progression in vitro and in vivo and could be reversed by miR145 restoration by mimetics. Also, GOLPH2 dependent metastasis was upregulated by mTOR downregulated miR-145 expression and was consequently also increased by miR-145 inhibitors. In this study the authors also show that GOLPH2 is found in exosomes, thereby spreading its tumourigenic properties to neighbouring cells.

Finally, another miRNA that apparently downregulates GOLPH2 is miR-200[33]. miR-200 is of special interest because its downregulation has been associated with EMT and invasion [34]. In adenocarcinoma of the lung miR-200 was found to be significantly downregulated resulting in high levels of GOLPH2. Restoration of miR-200 in cell lines and tumours attenuated the observed phenotype and showed the involvement of miR-200 and GOLPH2 in the pathogenesis of this cancer type.

The entity of the miRNA work underlines the role of GOLPH2 in malignant processes and demonstrates that cells regulate this protein tightly on different levels to avoid overexpression of GOLPH2 under physiologic circumstances.

\section{GOLPH2 is involved in several signalling pathways}

EGFR/RTK pathway: As mentioned, serum GOLPH2 is a potential biomarker in the diagnostics of HCC. Ye and colleagues first showed a close association of GOLPH2 upregulation with metastasis in HCC cohorts. In their study, they determined GOLPH2 to be a specific cargo adaptor assisting the epidermal growth factor receptor (EGFR), a member of receptor tyrosine kinases (RTKs), anchoring on the transGolgi network (TGN) and recycling back to plasma membrane in a ligand-dependent way [2]. These observations explain the proliferative properties of GOLPH2 via involvement in the EGFR pathway.

PDGFR pathway: Glioma cell lines expressing GOLPH2 show enhanced phosphorylation of AKT and ERK [35]. Further, invasion and migration were pronounced in these cell lines. These properties were reversed by GOLPH2 silencing through shRNA or at least partly reversed by the AKT inhibitor MK-2206. Gliomas of the proneural subtype, known to be often associated with PDGFRa gene alteration, expressed high levels of GOLPH2. The positive correlation of GOLPH2 and p-PDGFR $\alpha$ was confirmed by IHC of 29 GBM tissue samples. Further, AKT phosphorylation in response to PDGFA/PDGFRa signaling was abolished in the GOLPH2 silenced glioma cell line A172 indicating a potential key role of GOLPH2 in this pathway.

mTOR/Akt pathway: The most important downstream effector of EGFR/RTK is the mTOR complex. mTOR governs a wide variety of 
biological and cellular processes including cell growth, proliferation and survival. Aberrant mTOR pathway signaling has been observed in many types of cancers [36]. Recently, Gai and colleagues have shown that GOLPH2 activation of the mTOR pathway promotes proliferation and migration in HCC [32]. Their follow-up study of GOLPH2 and the mTOR interaction revealed miR-145 as a negative regulator of GOLPH2 to be a direct target of mTOR. Its downregulation was associated with GOLPH2 overexpression. An independent study from Chen and colleagues suggested that GOLPH2 is a novel effector of mTOR activation in hepatocellular carcinoma. Depletion of sGOLPH2 by a monoclonal antibody led to decreased cell proliferation [37]. The therapeutic potential of targeting GOLPH2 was successfully tested in a HCC xenograft model in nude mice.

GOLPH2 has been observed to foster prostate cancer progression mainly through the Phosphoinositide-3 Kinase (PI3K) Serine/ Threonine Kinase (AKT) mTOR signaling (PI3K-AKT-mTOR) [38]. PI3K inhibitor could abrogate the facilitative effect of GOLPH2 on cell proliferation and invasion in prostate cancer cell lines. On the other hand, overexpression of GOLPH2 promoted tumour growth in xenograft mice models of prostate cancer.

MEK/ERK pathway: Transforming growth factor- $\beta$ (TGF- $\beta$ ) functions as a tumour promotor during malignant progression. Yang and colleagues demonstrated that GOLPH2 is induced by TGF- $\beta$ and enhanced TGF- $\beta$-mediated extracellular signal regulated kinase (ERK) AKT activation (ERK/AKT). The ERK/AKT led to lipid raft expression that was higher in HCC in contrast to its inhibitory role on TGF- $\beta$ mediated Smad signaling [39]. This is in consistence with previous reports showing that GOLPH2 activates AKT signaling and modulates the signaling kinetics of EGFR/RTK complex recycling [2].

TLR/RIG-1 pathway: A study by Zhang et al. has identified GOLPH2 as a key negative regulator of innate immunity and by this supporting hepatitis $\mathrm{C}$ virus ( $\mathrm{HCV}$ ) infection. The interaction with mitochondrial anti-viral signaling proteins (MAVS) and TNF receptor associated factor 6 (TRAF6) as well as the MEK/ERK pathway are indispensable for this effect. After binding to MAVS and TRAF6, GOLPH2 promotes their degradation through a proteasome-dependent pathway [8]. The abolishment of these important proteins represses the host immune system and supports viral infection. Further influence of the host's immune system is mediated by GOPLH2's attenuation of the IFN- $\beta$ promoter, nuclear factor $\kappa \mathrm{B}(\mathrm{NF}-\mathrm{\kappa B})$ promoter and downregulation of IFN- $\beta$, interleukin-6 (IL-6) and other cytokines.

Taken together, abnormal GOLPH2 levels are involved in a variety of oncogenic signaling pathways in HCC, glioblastoma and prostate cancer. Thus, inhibition of GOLPH2 expression combined with a second target in EGFR/RTK, mTOR/AKT or MEK/ERK pathway could be a promising approach to control HCC, prostate and other cancer progression.

\section{GOLPH2 drives EMT}

An important step in carcinogenesis is the transition from an epithelial to mesenchymal phenotype. Thereafter, cells are equipped with higher abilities of migration and forming metastasis. Different groups have described the induction of EMT by GOLPH2 that could be reversed by consecutive ablation of the protein using for example siRNAs [1-3]. By targeting GOLPH2 this initiation of metastasis could possibly be interrupted resulting in better outcomes for cancer patients. In the respective animal models the researchers found exactly this correlation [2].

\section{Therapeutic targeting of GOLPH2}

There are few publications dealing with possibility of therapeutic targeting of GOLPH2. A gene therapy approach exploiting the herpes simplex virus thymidine kinase/ganciclovir (HSVtk/GCV) suicide gene system was conducted by Liu et al. in HCC [40]. In vitro and in vivo GP73-modified liposomes were successfully applied to target tumour tissues.

Another attempt comprised the design of an oncolytic adenovirus (GD55) where the GOLPH2 promotor is driving E1A gene expression. Cytotoxicity in tumour cells and growth inhibition in xenograft models of HCC [41,42] and prostate cancer [43] was achieved.

Li et al. published the construction of a mammalian cell-based display library and chose GOLPH2 as first target [44]. This approach would lead to direct panning of full-length antibodies. Further characterization of one of the anti-GOLPH2 antibodies has yet to be reported.

A conventional antibody for specific targeting: The cycling of GOLPH2 between the Golgi apparatus and the plasma membrane exposes the intralumenal part of GOLPH2 at the cell surface on tumour cells. When sGOLPH2 is cleaved close to the cell membrane, a short peptide is uncovered. This remnant peptide (rGOLPH2) marks GOLPH2 overexpressing and processing tumour cells. Specific antibodies against rGOLPH 2 have been developed and bind to GOLPH2 processing cells without being quenched by soluble GOLPH2 [45]. These antibodies are then internalized rapidly and offer themselves for antibody drug conjugate (ADC) delivery. Indeed, G2-2, a respective GOLPH2-ADC shows efficient inhibition of tumour cell proliferation in vitro and in vivo. In two patient derived xenograft models, a lung adeno carcinoma and a colorectal cancer model, G2-2-ADC showed efficient tumour growth inhibition and improved survival [45].

\section{Conclusion}

GOLPH2 has been suggested for cancer therapy by many researchers and various attempts in that direction have been tried. GOLPH2's predominantly intracellular localization and the extracellular shedding by proteolytic cleavage have so far precluded successful translation into clinical use. Methods that were successful in animal models like interfering RNA are not ready for clinical use yet. In this regard an antibody-based approach would be much more feasible. Antibodies with or without payloads have been successfully introduced for membrane-based antigens like EGFR or HER-2 into cancer therapy. However, in the case of GOLPH2 the cleavage and shedding of the protein poses a high obstacle to such an approach. The high concentration of soluble GOLPH2 in the serum and the surrounding tissue of the tumour cells would bind and neutralize the majority of therapeutic antibodies before they reach their actual destination. A novel antibody [45], G2-2, was recently developed to target the short portion of GOLPH2, that remains in the membrane after its cleavage. This approach might circumvent the problem of anti-GOLPH2 antibodies not reaching the target that other antibodies binding to the distal part of GOLPH2 would encounter.

It will be interesting to see how antibodies and other anti-GOLPH2 therapeutics will be further developed and what additional benefit targeting GOLPH2 will bring for cancer therapy. 


\section{References}

1. Bao Y (2013) Expression and prognostic significance of mesenchymal transition (EMT) related molecules in Hepatocellular Carcinoma (HCC). Diagn Pathol 8: 1-6.

2. Ye QH (2016) GOLM1 Modulates EGFR/RTK cell-surface recycling to drive hepatocellular carcinoma metastasis. Cancer Cell 30: 444-458.

3. Yang HJ, Liu GL, Liu B, Liu T (2018) GP73 promotes invasion and metastasis of bladder cancer by regulating the epithelial-mesenchymal transition through the TGF- $31 /$ Smad2 signalling pathway. J Cell Mol Med 22: 1650-1665.

4. Hu L, Li L, Xie H, Gu Y, Peng T, et al. (2011) The Golgi localization of GOLPH2 (GP73/GOLM1) is determined by the transmembrane and cytoplamic sequences. PLoS One 6: e28207. [Crossref]

5. Puri S, Bachert C, Fimmel CJ, Linstedt AD (2002) Cycling of early Golgi proteins via the cell surface and endosomes upon lumenal $\mathrm{pH}$ disruption. Traffic 3: 641-653. [Crossref]

6. Zhang W, Kim H, Lv J, Zhao N, Ma X, et al. (2018) Golgi Phosphoprotein 2 is a novel regulator of IL-12 production and macrophage polarization. J Immunol 200: 14801488. [Crossref]

7. Wright LM, Yong S, Picken MM, Rockey D, Fimmel CJ, et al. (2009) Decreased survival and hepato-renal pathology in mice with C-terminally truncated GP73 (GOLPH2). Int J Clin Exp Pathol 2: 34-47.

8. Zhang X (2017) GP73 represses host innate immune response to promote virus replication by facilitating MAVS and TRAF6 degradation. PLoS Pathog 13: 1-22.

9. Zhou Y, Yin X, Ying J, Zhang B (2012) Golgi protein 73 versus alpha-fetoprotein as a biomarker for hepatocellular carcinoma: a diagnostic meta- analysis. BMC Cancer 12: 17 .

10. Zhang F, Gu Y, Li X, Wang W, He J, et al. (2010) Up-regulated golgi phosphoprotein 2 (GOLPH2) expression in lung adenocarcinoma tissue. Clin Biochem 43: 983-991. [Crossref]

11. Marrero JA (2005) GP73, a resident Golgi glycoprotein, is a novel serum marker for hepatocellular carcinoma. J Hepatol 43: 1007-10012.

12. Riener MO (2009) Golgi phosphoprotein 2 (GOLPH2) expression in liver tumors and its value as a serum marker in hepatocellular carcinomas. Hepatology 49: 1602-1609.

13. Tan L (2009) Correlaion between serum Golph2 protein and hepatocellular carcinoma. Zhonghua Gan Zang Bing Za Zhi 17: 288-291.

14. Ju Q (2013) Monoclonal antibody preparation of golgi phosphoprotein 2 and preliminary application in the early diagnosis of hepatocellular carcinoma. Mol Med Rep 8: 517-522.

15. Li B (2017) The clinical values of serum markers in the early prediction of hepatocellular carcinoma. Biomed Res Int 2017: 5358615 [Crossref]

16. Kristiansen G (2008) GOLPH2 protein expression as a novel tissue biomarker for prostate cancer: implications for tissue-based diagnostics. Br J Cancer 99: 939-948 [Crossref]

17. Liu G, Zhang Y, He F, Li J, Wei X, et al. (2014) Expression of GOLPH2 is associated with the progression of and poor prognosis in gastric cancer. Oncol Rep 32: 2077-2085. [Crossref]

18. Zhang F (2010) Up-regulated golgi phosphoprotein 2 (GOLPH2) expression in lung adenocarcinoma tissue. Clin Biochem 43: 983-991.

19. Zhang Y (2017) Association of GOLPH2 expression with survival in non-small-cell lung cancer: clinical implications and biological validation. Biomark Med 11: 967-977.

20. Ding X (2019) GOLM1 silencing inhibits the proliferation and motility of human glioblastoma cells via the Wnt/ß-catenin signaling pathway. Brain Res 1717: 117-126.

21. Donizy P (2016) Golgi-related proteins GOLPH2 (GP73/GOLM1) and GOLPH3 (GOPP1/MIDAS) in cutaneous melanoma: patterns of expression and prognostic significance. Int J Mol Sci 17: 1619.

22. Riener MO (2009) Golgi phosphoprotein 2 (GOLPH2) expression in liver tumors and its value as a serum marker in hepatocellular carcinomas. Hepatology 49: 1602-1609 [Crossref]

23. Shan SG (2013) Gradually increased Golgi protein 73 expression in the progression of benign liver diseases to precancerous lesions and hepatocellular carcinoma correlates with prognosis of patients. Hepatol Res 43: 1199-1210.

24. Liu T (2017) Serum Golgi protein 73 is not a suitable diagnostic marker for hepatocellular carcinoma. Oncotarget 8: 16498-16506. [Crossref]

25. Laxman B (2008) A first-generation multiplex biomarker analysis of urine for the early detection of prostate cancer. Cancer Res 68: 645-649.
26. Varambally S, Laxman B, Mehra R, Cao Q, Dhanasekaran SM, et al. (2008) Golgi protein GOLM1 is a tissue and urine biomarker of prostate cancer. Neoplasia 10: 12851294. [Crossref]

27. Wei S, Dunn TA, Isaacs WB, De Marzo AM, Luo J (2008) GOLPH2 and MYO6: putative prostate cancer markers localized to the Golgi apparatus. Prostate 68: 13871395.

28. Laxman B, Morris DS, Yu J, Siddiqui J, Cao J, et al. (2008) A first-generation multiplex biomarker analysis of urine for the early detection of prostate cancer. Cancer Res 68: 645-649. [Crossref]

29. Liu X, Chen L, Zhang T (2018) Increased GOLM1 Expression independently predicts unfavorable overall survival and recurrence-free survival in lung adenocarcinoma. Cancer Control 25: 1073274818778001.

30. Li H, Yang LL, Xiao Y, Deng WW, Chen L, et al. (2018) Overexpression of golg phosphoprotein 2 is associated with poor prognosis in oral squamous cell carcinoma. $\mathrm{Am}$ J Clin Pathol 150: 74-83. [Crossref]

31. Zhou M, Chen X, Wu J, He X, Ren R (2018) MicroRNA-143 regulates cell migration and invasion by targeting GOLM1 in cervical cancer. Oncol Lett 16: 6393-6400. [Crossref]

32. Gai X (2019) mTOR/miR-145-regulated exosomal GOLM1 promotes hepatocellular carcinoma through augmented GSK-33/MMPs. J Genet Genomics 46: 235-245.

33. Yang L, Luo P, Song Q, Fei X (2018) DNMT1/miR-200a/GOLM1 signaling pathway regulates lung adenocarcinoma cells proliferation. Biomed. Pharmacother 99: 839-847.

34. Paterson EL (2013) Down-Regulation of the miRNA-200 family at the invasive front of colorectal cancers with degraded basement membrane indicates emt is involved in cancer progression. Neoplasia 15: 180-IN22.

35. Xu R (2017) PDGFA/PDGFRa-regulated GOLM1 promotes human glioma progression through activation of AKT. $J$ Exp Clin Cancer Res 36: 1-17.

36. Mossmann D, Park S, Hall MN (2018) mTOR signalling and cellular metabolism are mutual determinants in cancer. Nat Rev Cancer 18: 744-757. [Crossref]

37. Chen X (2015) MTORC1 up-regulates GP73 to promote proliferation and migration of hepatocellular carcinoma cells and growth of xenograft tumors in mice. Gastroenterology 149: 741e14-752e14.

38. Yan G (2017) GOLM1 promotes prostate cancer progression through activating PI3KAKT-mTOR signaling. Prostate 1-12.

39. Yang X (2013) GP73, a novel TGF- $\beta$ target gene, provides selective regulation on Smad and non-Smad signaling pathways. Biochim Biophys Acta Mol Cell Res 1866: 588-597.

40. Liu C (2019) golgi membrane protein GP73 modified-liposome mediates the antitumor effect of survivin promoter-driven HSVtk in hepatocellular carcinoma. Exp Cell Res 111496.

41. Wang Y (2015) A novel golgi protein (GOLPH2)-regulated oncolytic adenovirus exhibits potent antitumor efficacy in hepatocellular carcinoma. Oncotarget 6: 1356413578.

42. Zhang X (2017) GP73-regulated oncolytic adenoviruses possess potent killing effect on human liver cancer stem-like cells. Oncotarget 7: 29346-29358

43. Ying C (2017) GOLPH2-regulated oncolytic adenovirus, GD55, exerts strong killing effect on human prostate cancer stem-like cells in vitro and in vivo. Nat Publ Gr 39: 405-414.

44. Li F (2012) Construction and development of a mammalian cell-based full-length antibody display library for targeting hepatocellular carcinoma. Appl Microbiol Biotechnol 96: 1233-1241.

45. Liewen H (2019) Therapeutic targeting of golgi phosphoprotein 2 (GOLPH2) with Armed Antibodies: A Preclinical Study of Anti-GOLPH2 antibody drug conjugates in lung and colorectal cancer models of patient derived xenografts (PDX). Target Onco 14: 577-590. [Crossref]

Copyright: (C2019 Liu Y. This is an open-access article distributed under the terms of the Creative Commons Attribution License, which permits unrestricted use, distribution, and reproduction in any medium, provided the original author and source are credited. 